\title{
Nanopipes in GaN: photo-etching and TEM study
}

\author{
S. Lazar ${ }^{1, a}$, J. L Weyher ${ }^{2,3}$, L. Macht ${ }^{2}$, F. D. Tichelaar ${ }^{1}$, and H. W. Zandbergen ${ }^{1}$ \\ 1 National Center for HREM, Kavli Institute of Nanoscience Delft University of Technology, Rotterdamseweg 137, \\ 2628 AL Delft, The Netherlands \\ 2 Experimental Solid State Physics III, RIM, University of Nijmegen, Toernooiveld 1, 6525 ED Nijmegen, The Netherlands \\ 3 High Pressure Research Center, Polish Academy of Sciences, Sokolowska 29/37, 01-142 Warsaw, Poland
}

Received: 19 June 2003 / Accepted: 28 January 2004 - (C) EDP Sciences

\begin{abstract}
Photochemical (PEC) etching and transmission electron microscopy (TEM) have been used to study the defects in hetero-epitaxial GaN layers. TEM proved that PEC etching reveals not only dislocations but also nanopipes in the form of protruding, whisker-like etch features. It is shown by diffraction contrast techniques that the nanopipes are screw coreless dislocations. An example is shown of the transformation of a normal full-core screw dislocation into a nanopipe. The PEC/TEM experiments indicate the presence of electrically active (recombinative) species in the vicinity of the nanopipes.
\end{abstract}

PACS. 61.72.Ff Direct observation of dislocations and other defects (etch pits, decoration, electron microscopy, x-ray topography, etc.)

\section{Introduction}

In spite of high density of defects in hetero-epitaxial layers, GaN has been used with a surprising success in production of high efficiency LEDs and laser diode with a short wavelength in the visible region of the spectrum. In order to understand the nature and the properties of defects in nitrides numerous theoretical and physical studies were undertaken in the last decade. Various types of structural defects were reported and characterized in GaN layers, namely threading dislocations (TDs), inversion domains (IDs), nanopipes and pinholes [1-6]. In particular TEM is used to determine the nature of these defects, but also various defect-selective etching methods have been used successfully for revealing and analyzing the defects in GaN [7-12]. Amongst these methods photoelectrochemical (PEC) etching [10] seems to be very promising. The reliability of PEC method in revealing dislocations in GaN has been confirmed by direct TEM calibration $[10,13]$ and compared with the results of "orthodox" etching [14]. In addition it was shown that the method is capable of revealing IDs in the form of tiny whiskers similar to those formed on dislocations or in the form of deep craters, depending upon their diameter $[15,16]$.

Recently, using defect-selective "orthodox" etching and TEM on Ga-polar GaN hetero-epitaxial layers, we observed - apart from threading dislocations - also nanopipes [12]. The density of nanopipes remains two orders of magnitude lower than that of dislocations, i.e. is about $2 \times 10^{7} \mathrm{~cm}^{-2}$. In this communication the results are

\footnotetext{
a e-mail: S.Lazar@tnw.tudelft.nl
}

reported on the cross-sectional TEM examination of PECetched Ga-polar hetero-epitaxial GaN layers (the same material as in [12]) with the aim of finding a correlation between nanopipes and the etch features. The character and the origin of nanopipes are also investigated.

\section{Experiments and results}

The samples were grown by metal-organic chemical-vapor deposition (MOCVD) on sapphire substrates following the procedure described earlier [17]. Standard wurtzite-type Ga-polar GaN was grown on a 20 nm-thick GaN buffer layer. PEC etching was performed using stirred $0.004 \mathrm{M}$ $\mathrm{KOH}$ solution, Ti on GaN contacts and $450 \mathrm{~W}$ Xe lamp illumination. In order to avoid breaking of defect-related whisker-like etch features during preparation of the thin foils, the GaN samples were etched only for 5 minutes. After etching, the samples were examined in scanning electron microscope (SEM) and subsequently, cross-sectional electron transparent foils were prepared by mechanical thinning followed by Ar-ion milling. Thin foils were examined in a Philips CM30T microscope operating at $300 \mathrm{kV}$. Diffraction contrast images were obtained in dark field using two-beam conditions, or with reflections limited to a systematic row. In order to have a good spatial resolution of the core dislocations the images were recorded under weak beam imaging conditions.

A typical surface morphology of PEC-etched GaN sample is shown in Figure 1. The whole surface is covered by a high density of straight whiskers, all about $50-60 \mathrm{~nm}$ in diameter. Conventional diffraction contrast 


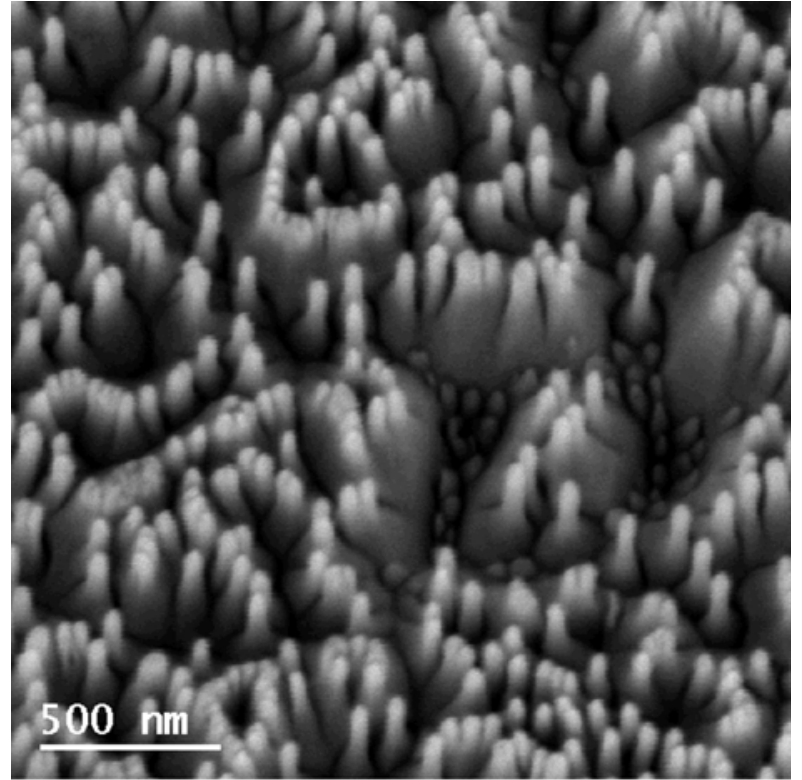

Fig. 1. SEM image of Ga-polar hetero-epitaxial GaN layer after PEC etching in $\mathrm{KOH}$ solution. Sample tilted $45^{\circ}$.
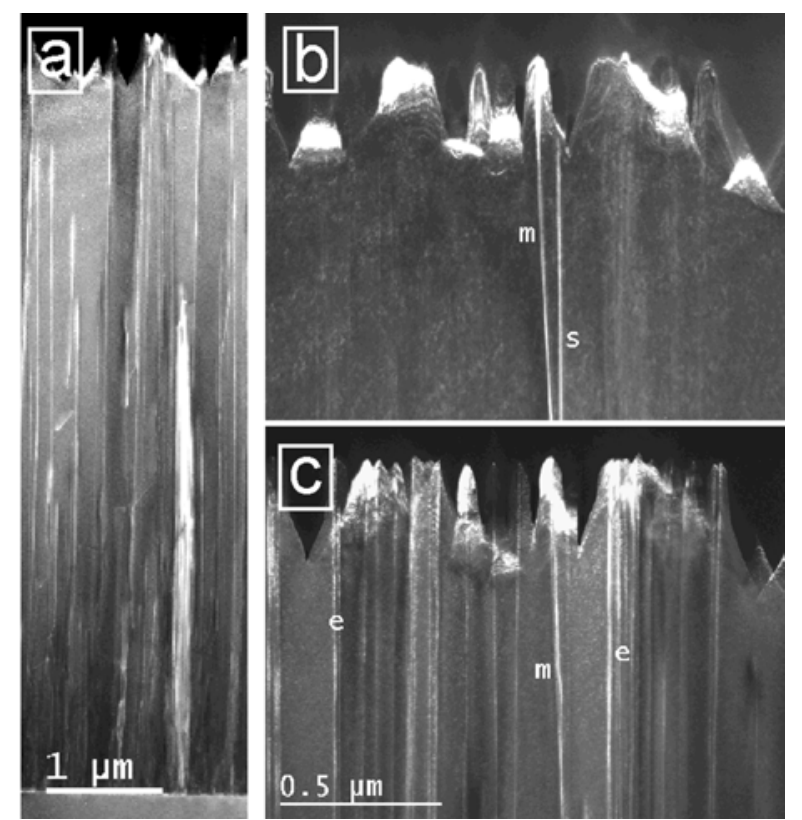

Fig. 2. Weak beam dark field cross-sectional TEM images of PEC-etched specimen $\sim g(3 g)$, (a) whole epilayer with $g=$ $11 \overline{2} 0$, (b) with $\mathrm{g}=0002$, (c) with $g=11 \overline{2} 0$. The images show the correlation between whiskers and mixed $(\mathrm{m})$, screw $(\mathrm{s})$ and edge (e) dislocations.

analysis of the cross section of this GaN layer revealed, that a vast majority of the whisker-like etch features were formed on dislocations as is demonstrated in Figure 2. The dislocations are straight and threading across the whole thickness of the layer (Figure $2 \mathrm{a}$ ). This observation remains in agreement with the data published earlier [15]. The analysis with the invisibility criterion for dislocation $g^{*} b=0$ shows that most of the dislocations have a mixed
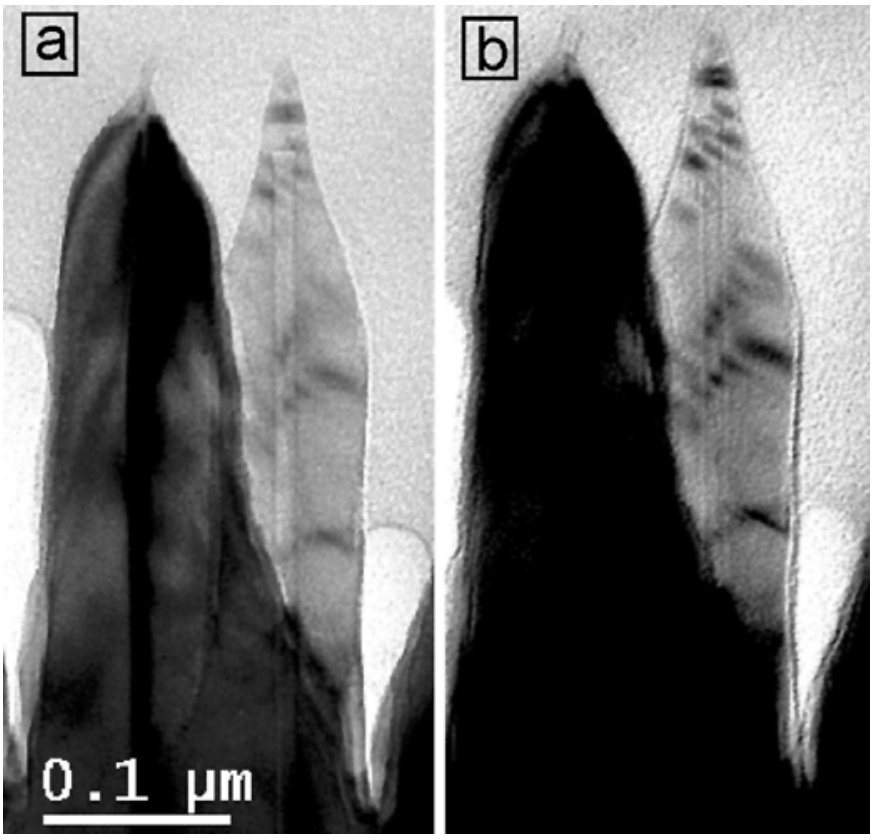

Fig. 3. Fresnel contrast images of a nanopipe in two beam condition with $g=0002$ excited close to [1100] zone axis. (a) Under-focused image, (b) over-focused image.

$(b=1 / 3\langle 11 \overline{2} 3\rangle)$ or edge $(b=1 / 3\langle 11 \overline{2} 0\rangle)$ character but also some pure screw dislocations (about $3 \%$ of the whole population of dislocations) were encountered (Figure $2 \mathrm{~b}-$ c). It has been found that the whisker-like etch features are formed on all three types of dislocations, which shows that all defects are electrically active (recombinative for photo-carriers).

Detailed TEM inspection of the specimens showed also the presence of another type of defects, namely hollow tubes. These defects are known as nanopipes [2-6] (coreless or open-core dislocations). In Figure 3 an example of a whisker formed on a nanopipe is shown. In order to enhance the visibility the images were taken in defocused conditions i.e. Fresnel contrast conditions [18]. Due to the difference of inner potential between matrix $(\mathrm{GaN})$ and the core of the nanopipe (vacuum) we can see that in under-focused image the core of the nanopipe is bright surrounded by dark fringes and in over-focused image the core is dark surrounded by bright fringes. The nanopipes which were found in this specimen have a diameter in the range of 8-12 $\mathrm{nm}$ and a length along the c axis about $1 \mu \mathrm{m}$. All of them have a uniform diameter through-out the layer and most of them have funnel-shaped outcrops. In [6] nanopipes with a diameter in the range of 2-40 nm and a length along the $\mathrm{c}$ axis between $10-400 \mathrm{~nm}$ were reported.

In order to find out the origin and the character of the nanopipes revealed by PEC etching, TEM images with different excited reflections were taken. The image in Figure $4 \mathrm{~b}$ was taken with $g=0002$ excited, resulting visibility of mixed and screw dislocations only. The segment $\mathbf{m}$, that is a mixed type dislocation with Burgers vector $b=1 / 3\langle 11 \overline{2} 3\rangle$, propagated from the substrate/GaN 

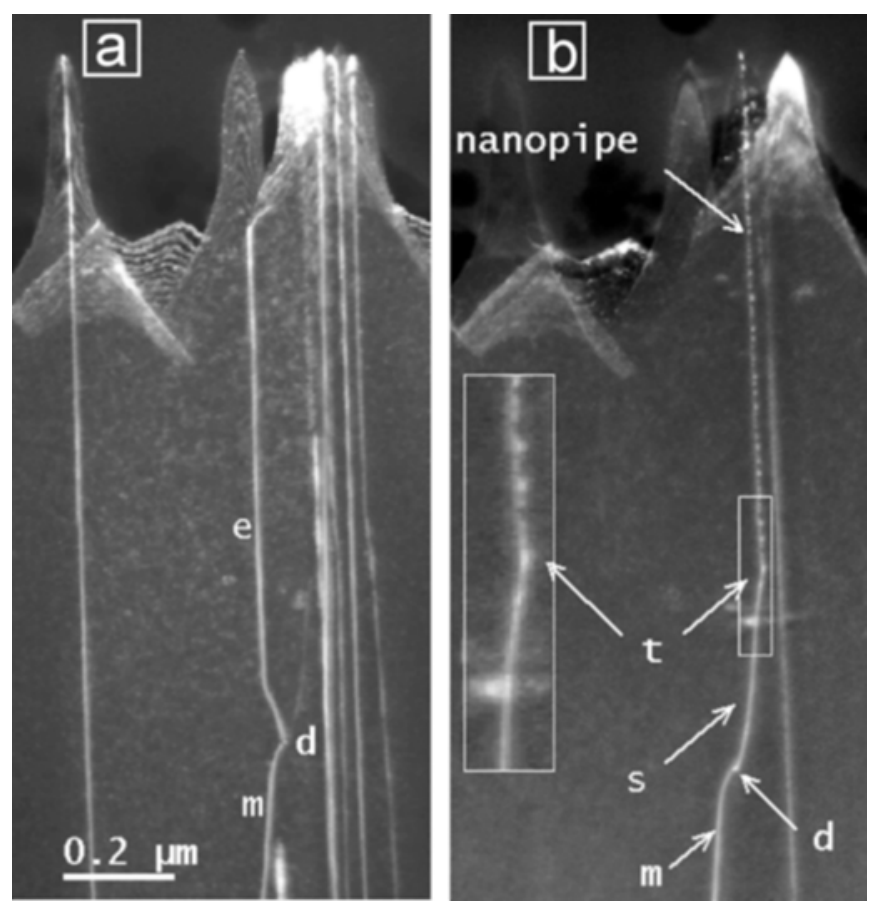

Fig. 4. Weak beam dark field images $\sim g(3 g)$ close to $[1 \overline{1} 00]$ zone axis (a) with $g=11 \overline{2} 0$, (b) with $g=0002$. The images show the dissociation reaction of a mixed type dislocation in an edge dislocation (e) and a screw one (s). The screw dislocation becomes a nanopipe at the point $\mathbf{t}$.

layer interface through the layer and dissociated at the position marked $\mathbf{d}$ into a screw dislocation (the segment $\mathbf{s}$ in Figure $4 \mathrm{~b}$ ) with Burgers vector $b=[0001]$ and an edge dislocation (the segment e in Figure 4 a) according to the reaction:

$$
1 / 3\langle 11 \overline{2} 3\rangle \rightarrow 1 / 3\langle 11 \overline{2} 0\rangle+\langle 0001\rangle .
$$

Although this reaction is energetically neutral, it may occur because of local strain conditions [19]. At a certain point ( $\mathbf{t}$ in Figure $4 \mathrm{~b}$ ) the screw dislocation (the segment $\mathbf{s}$ ) transformed into a nanopipe, which propagates further along the $\mathbf{c}$ direction to the surface of the epitaxial layer. The transformation point where the screw dislocation became a nanopipe with a perfect screw character (Figure $4 \mathrm{~b}$ ) can be observed due to the change of contrast. Since the weak beam contrast is sensitive to the displacements on a short-range distance, the displacement vector may vary along the nanopipe giving rise to the granular contrast in Figure $4 \mathrm{~b}$. The contrast of the nanopipe is given by the side walls, not by the hollow tube itself. Therefore, different degree of decoration of the side walls of the nanopipes could be also responsible for this variable contrast. Using LACBED technique [5], which is sensitive to long range displacements, it was shown that most of the nanopipes are hollow core screw dislocations with Burgers vectors $\mathbf{c}$ or $-\mathbf{c}$.

There are both theoretical and experimental data on the role of oxygen in the formation of nanopipes and on the effect of segregation of oxygen to form stable $\mathrm{V}_{\mathrm{Ga}^{-}}\left(\mathrm{O}_{\mathrm{N}}\right)_{3}$ complexes at the walls of the nanopipes [20,21]. These specific complexes are electrically inert [21], therefore they should not contribute to the recombination of photogenerated carriers during PEC etching. However, there are other types of oxygen- or Ga-related defects, which are electrically active [22], and which might be formed in the strain field of screw dislocations or at the nanopipe side walls. These defects (called atmosphere in related papers) could contribute to the recombination of photo-generated carriers leading to the formation of the protruding etch features. Because the size and diameter of whiskers formed on nanopipes (see Figure 3) is comparable to those of dislocations during PEC etching, the recombinative effectiveness of such atmosphere on nanopipes is similar to that of dislocations.

\section{Conclusions}

In conclusion, we have shown that PEC etching reveals in GaN hetero-epitaxial layers all types of dislocations (edge, mixed and screw) and nanopipes in the form of protruding, whisker-like etch features which means that all these defects are electrically active. From SEM observation of the etched surface it is not possible to distinguish dislocations from nanopipes. Similar morphology of the etch features suggests similar recombinative effectiveness of all these defects, including the nanopipes, for the photo-generated carriers. The nanopipes, which we found in the thin foil, were associated with coreless screw type dislocations. An example was shown of the "transformation" of a normal screw dislocation into a nanopipe, which leads to the conclusion that the common full core dislocations are among the precursors in the formation $[4,6]$ of the nanopipes. The origin of the "transformation" of a normal screw dislocation into a nanopipe is unknown. Further work is planned in order to establish the presence and types of electrically active defects in the vicinity of the nanopipes.

This work is part of the research programme of the 'Stichting voor Fundamenteel Onderzoek der Materie (FOM)', which is financially supported by the 'Nederlandse Organisatie voor Wetenschappelijk Onderzoek (NWO)'.

\section{References}

1. F. A. Ponce, D. Cherns, W. T. Young, J. W. Steeds, Appl. Phys. Lett. 69, 770 (1997)

2. W. Qian, M. Skowronski, K. Doverspike, L. B. Rowland, D. K. Gaskill, J. Cryst. Growth 151, 396 (1995)

3. D. Cherns, W. T. Young, F. A. Ponce, Mater. Sci. Eng. B 50, 76 (1997)

4. P. Vennegues, B. Beaumont, M. Vaille, P. Gibart, Appl. Phys. Lett. 70, 2434 (1997)

5. D. Cherns, W. T. Young, J. W. Steeds, F. A. Ponce, S. Nakamura, J. Cryst. Growth 178, 201 (1997) 
6. Z. Liliental-Weber, Y. Chen, S. Ruvimov, J. Washburn, Phys. Rev. Lett. 79, 2835 (1997)

7. K. Shiojima, J. Vac. Sci. Technol. B 18, 37 (2000)

8. S. H. Hong, B. J. Kim, H. S. Park, Y. Park, S. Y. Yoon, T. I. Kim, J. Cryst. Growth 191, 275 (1998)

9. M. Iwaya, T. Takeuchi, S. Yamaguchi, C. Wetzel, H. Amano, I. Akasaki, Jpn J. Appl. Phys. 37, L316 (1998)

10. C. Youtsey, L. T. Romano, I. Adesida, Appl. Phys. Lett. 73, 797 (1998)

11. J. L. Weyher, P. D. Brown, J. L. Rouviere, T. Wosinski, A. R. A. Zauner, I. Grzegory, J. Cryst. Growth 210, 151 (2000)

12. G. Kamler, J. L. Weyher, I. Grzegory, E. Jezierska, T. Wosinski, J. Cryst. Growth 246, 21 (2002)

13. C. Youtsey, L. T. Romano, R. J. Molnar, I. Adesida, Appl. Phys. Lett. 74, 3537 (1999)

14. P. Visconti, D. Huang, M. A. Reshchikov, F. Yun, R. Cingolani, D. J. Smith, J. Jasinski, W. Swider, Z. Liliental-Weber, H. Morkoç, Mater. Sci. Eng. B 93, 229 (2002)

15. J. L. Weyher, F. D. Tichelaar, H. W. Zandbergen, L. Macht, P. R. Hageman, J. Appl. Phys. 90, 6105 (2001)
16. J. L. Weyher, L. Macht, F. D. Tichelaar, H. W. Zandbergen, P. R. Hageman, P. K. Larsen, Mater. Sci. Eng. B 91-92, 280 (2002)

17. F. K. de Theije, A. R. A. Zauner, P. R. Hageman, W. J. P. van Enckevort, P. K. Larsen, J. Cryst. Growth 197, 37 (1999)

18. D. B. Williams, C. B. Carter, Transmission Electron Microscopy textbook for material sciences (Plenum Press, New York, 1996)

19. F. R. Chien, X. J. Ning, S. Stemmer, P. Pirouz, M. D. Bremser, R. F. Davis, Appl. Phys. Lett. 68, 2678 (1996)

20. S. Ruvimov, Z. Liliental-Weber, C. Dieker, J. W. M. Koike, H. Amano, I. Akasaki, Mat. Res. Soc. Symp. Proc. 468, 287 (1997)

21. J. Elsner, R. Jones, M. Haugk, R. Gutierrez, Th. Frauenheim, S. Öberg, P. R. Briddon, Appl. Phys. Lett. 73, 3530 (1998)

22. J. Elsner, A. Th. Blumenau, Th. Frauenheim, R. Jones, M. I. Heggie, MRS Internet J. Nitride Semicond. Res. 5S1, W9.3 (2000) 